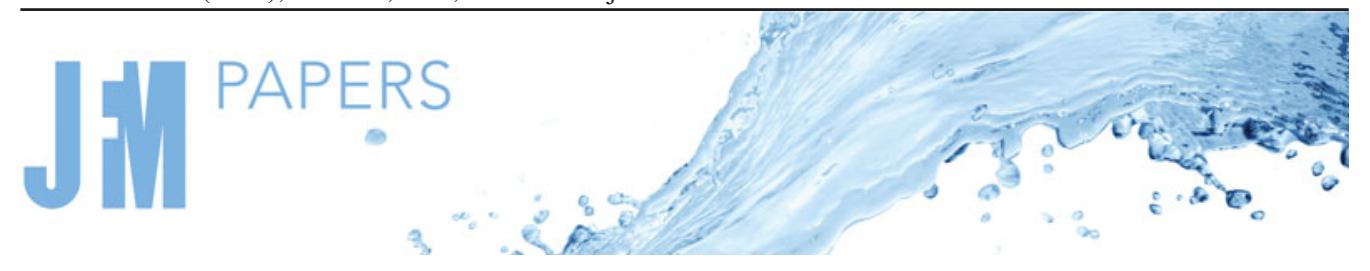

\title{
High-Reynolds-number wake of a slender body
}

\author{
J.L. Ortiz-Tarin ${ }^{1}$, S. Nidhan ${ }^{1}$ and S. Sarkar ${ }^{1} \uparrow$ \\ ${ }^{1}$ Department of Mechanical and Aerospace Engineering, University of California San Diego, \\ CA 92093, USA
}

(Received 2 December 2020; revised 18 February 2021; accepted 16 April 2021)

The high-Reynolds-number axisymmetric wake of a slender body with a turbulent boundary layer is investigated using a hybrid simulation. The wake generator is a $6: 1$ prolate spheroid and the Reynolds number based on the diameter $D$ is $\operatorname{Re}=10^{5}$. The transition of the wake to a state of complete self-similarity is investigated by looking for the first time into the far field of a slender-body wake. Unlike bluff-body wakes, here the flow is not dominated by vortex shedding in the near wake. Instead, the recirculation region is very small, the near wake is quasi-parallel and is characterised by the presence of broadband turbulence. Until $x / D \approx 20$, the wake decay of a slender body with turbulent boundary layer is very similar to the classic high- $R e$ behaviour, $U_{d} \sim x^{-2 / 3}$. Extrapolation of this observation to larger $x / D$ has led to the belief that these wakes decay following the asymptotic $-2 / 3$ decay law. Our results show, however, that this is not the case and the wake transitions to a faster decay rate once complete self-similarity is achieved. In this later region $(20<x / D<80)$, mean and turbulence profiles are self-similar. Furthermore, despite the high global and local Reynolds numbers, the classic hypotheses that lead to the well-known decay exponents are not fulfilled. Instead, turbulent dissipation follows a non-equilibrium scaling and a new decay rate $U_{d} \sim x^{-6 / 5}$ is observed. The transition from $U_{d} \sim x^{-2 / 3}$ to $U_{d} \sim x^{-6 / 5}$ is preceded by the dominance of the azimuthal $|m|=1$ mode and the emergence of a large-scale helical structure.

Key words: wakes, shear layer turbulence

\section{Introduction}

Turbulent wakes are ubiquitous in nature. From the wind fluctuations generated by buildings to the large-scale oceanic structures left by currents flowing past islands, turbulent wakes distribute energy and momentum across space in a wide variety of situations and environments. In this work, we focus on the wake generated by

$†$ Email address for correspondence: sarkar@ucsd.edu

(C) The Author(s), 2021. Published by Cambridge University Press. This is an Open Access article, distributed under the terms of the Creative Commons Attribution licence (http://creativecommons.org/ licenses/by/4.0/), which permits unrestricted re-use, distribution, and reproduction in any medium, provided the original work is properly cited. 


\section{J.L. Ortiz-Tarin, S. Nidhan and S. Sarkar}

an axisymmetric slender body, which is particularly relevant for aerodynamic and hydrodynamic applications.

As a canonical shear flow that can serve as a building block to study more complex flows, turbulent wakes have been studied extensively in the past. However, an overall wake decay theory is still missing and recent studies have shown a richer and more complex problem than that portrayed by classical theory. The classic solutions for the decay of the axisymmetric turbulent wake assume that the flow evolves in a self-similar manner (Tennekes \& Lumley 1972; Townsend 1976). They are based on the hypothesis that the profiles of the velocities and the Reynolds stresses become invariant with respect to $x$ (the streamwise coordinate) when expressed in terms of local characteristic scales. The self-similarity concept is often combined with the notion of universality (Townsend 1976). According to the universality hypothesis, far from their generators, all wakes evolve into a universal state, where the chaotic mixing of eddies has erased the memory of the initial conditions. In the self-similar and universal state, an axisymmetric wake obeys a single set of power-law exponents. The classic high-Reynolds-number wake laws are $U_{d} \sim x^{-2 / 3}$ and $L \sim x^{1 / 3}$ where $U_{d}$ is the centreline velocity deficit and $L$ is the wake width. At low Reynolds number, the laws change to $U_{d} \sim x^{-1}$ and $L \sim x^{1 / 2}$. In the last decades, the classical decay laws together with evidence of universality have remained elusive to experiments and simulations. Studying turbulent wakes is challenging, it requires measuring weak fluctuations in extremely long domains, at high Reynolds numbers, and during time intervals which are sufficiently long to reach statistical convergence. This has led to some contradictory and ambiguous evidence regarding the wake evolution.

The early experiments of Carmody (1964) employed a disk as a wake generator. At $R e=7 \times 10^{4}$ and in a domain up to $x / D=15$, he found $U_{d} \sim x^{-2 / 3}$. Some years later, Chevray (1968) reported the first experiment on a high-Re slender-body wake. Using a 6: 1 prolate spheroid with a tripped boundary layer, he also observed $U_{d} \sim x^{-2 / 3}$. This experiment was at $R e=4.5 \times 10^{5}$ and spanned a downstream distance of $18 \mathrm{D}$. Around the same time, Gibson, Chen $\&$ Lin (1968) using a sphere at $R e=6.5 \times 10^{4}$ measured a sharper decay, $U_{d} \sim x^{-0.85}$ (between classic low- and high-Re laws) up to $x / D \approx 60$. The studies of Uberoi \& Freymuth (1970) and Bevilaqua \& Lykoudis (1978) report $U_{d} \sim x^{-2 / 3}$ in the lee of a sphere at $R e=8.6 \times 10^{3}$ and $R e=10^{4}$, respectively. However, Uberoi \& Freymuth (1970) only observed the $-2 / 3$ decay after $x / D=50$ in a domain that spanned $x / D=300$ whereas Bevilaqua \& Lykoudis (1978) found it after $x / D=8$ in a domain that spanned until $x / D=100$. In both cases, the sphere wakes had local Re below 500. This is a threshold that, years later, Johansson, George \& Gourlay (2003) would suggest as the limit below which a wake starts showing a low-Re $U_{d} \sim x^{-1}$ decay. George (1989) is the first work that emphasises that the classical way of looking at wake power laws may be oversimplified and introduces the idea of local and partial self-similarity conditions. Two years later, Cannon (1991) performed an extensive series of experiments looking at the evolution of the far wake with different wake generators. He found that a fully self-similar state was not achieved in a domain that spanned $x / D \approx 100$ at Reynolds numbers $\operatorname{Re}_{D}=$ $1.3-2.15 \times 10^{4}$. His work pointed to the existing ambiguity between the high- and low-Re decay laws and to the necessity of an additional characteristic velocity, apart from $U_{d}$, to scale the results. In a later study, Bonnier \& Eiff (2002) reported $U_{d} \sim x^{-0.97}$ for a sphere wake at $\operatorname{Re}$ up to $1.2 \times 10^{4}$.

With the advent of increasing computational power, the use of temporal simulations enabled for the first time the analysis of the very far wake, up to $x / D \sim O\left(10^{3}\right)$. Temporal simulations make use of the Galilean transformation that relates time in a reference frame moving with the wake to the streamwise distance in a fixed reference frame $t=x / U$, where $U$ is the velocity of the body. By assuming that the downstream development of flow 


\section{High-Reynolds-number wake of a slender body}

statistics is slow, the temporal simulations use streamwise periodic boundary conditions and advance the equations in time. Instead of introducing the wake generator, an initial condition is chosen to approximate the flow at some distance from the body. The use of a temporal model reduces the computational cost very significantly but requires a good initial condition to achieve a quantitative match with experiments. Expanding on the results of Gourlay et al. (2001), Johansson et al. (2003) showed that the rate of decay of the wake might depend on the local Reynolds number. In their computational study, the wake transitioned from a decay rate of $-2 / 3$ to -1 when the value of the local Reynolds number, $R e_{L}=U_{d} L / v$, went below approximately 500. In a later temporal study, Redford et al. (2012) performed two temporal simulations with different initial conditions. They showed that universality was achieved only after a long passage of time, and suggested that the universal regime might not be seen in practice. Interestingly, in the far wake, the flow statistics followed the high-Re decay despite the local $R e$ falling below 500 .

One year later, Nedić et al. (2013) introduced the concept of non-equilibrium dissipation to the study of axisymmetric wakes. Instead of assuming that turbulent dissipation scales as $\varepsilon \sim k^{3 / 2} / L$, where $k$ is the turbulent kinetic energy (TKE) and $L$ is a characteristic large-eddy length, a new scaling $\varepsilon \sim\left(\operatorname{Re} / \operatorname{Re}_{L}\right)^{n} k^{3 / 2} / L$ was proposed. This non-equilibrium dissipation scaling has been attributed to unsteadiness of the energy cascade and has led to a re-examination of the classical decay exponents. For example, the experiments and simulations of Dairay et al. (2015) found that the wake of a $R e=5 \times 10^{3}$ fractal plate exhibits a $U_{d} \sim x^{-1}$ non-equilibrium decay. According to classic theory, this wake should have decayed as $x^{-2 / 3}$ because $R e_{L}$ was high. However, this was not the case and only the non-equilibrium theory was able to explain it. Other works such as Obligado, Dairay \& Vassilicos (2016) and more recently Chongsiripinyo \& Sarkar (2020) have also observed this non-equilibrium region in the wake of square plates and disks.

Despite the use of slender bodies in many engineering applications, the majority of wake studies are devoted to the wake of bluff bodies. There are some particularities that make the study of high-Reynolds-number slender-body wakes especially challenging. Computationally, it is very costly to simulate turbulent boundary layers over long bodies and also resolve their far wake. The problem is tremendously stiff and the required spatial and temporal resolution is enormous. In a wind tunnel or in a tank, if the body is long, a significant amount of the measuring section can be taken by the wake generator. Furthermore, slender-body wakes are thinner than bluff-body wakes and potentially harder to probe and measure. These constraints have significantly limited the available studies on the topic.

The first study of this kind is Chevray (1968). In his work, a $6: 1$ prolate spheroid with a tripped boundary layer was used as a wake generator. The Reynolds number based on the diameter was $R e=4.5 \times 10^{5}$ and the domain spanned $x / D=18$. In this region, the wake defect velocity showed a decay of $U_{d} \sim x^{-2 / 3}$ and self-similarity was achieved only in the mean velocity. Years later, Jiménez et al. (2010) performed a study of the near wake of a standard submarine model (DARPA SUBOFF) with a tripped boundary layer at $R e=$ $1.2 \times 10^{5}-7.7 \times 10^{5}$. This study, spanning 15 diameters, also showed the wake decay following $U_{d} \sim x^{-2 / 3}$. Only recently, high-resolution body-inclusive (BI) simulations have provided more data on the decay of the slender-body axisymmetric wake. Posa $\&$ Balaras (2016) observed a $-2 / 3$ decay for the wake of a SUBOFF at $R e=1.39 \times 10^{5}$ in a domain up to $x / D=12$. Kumar $\&$ Mahesh (2018) simulated flow past an unappended SUBOFF at

$R e=1.26 \times 10^{5}$ in a domain that spanned $x / D=15$. They observed $-2 / 3$ decay followed by a transition to -1 at $x / D \approx 5$, which they attributed to low-Re decay. 


\section{J.L. Ortiz-Tarin, S. Nidhan and S. Sarkar}

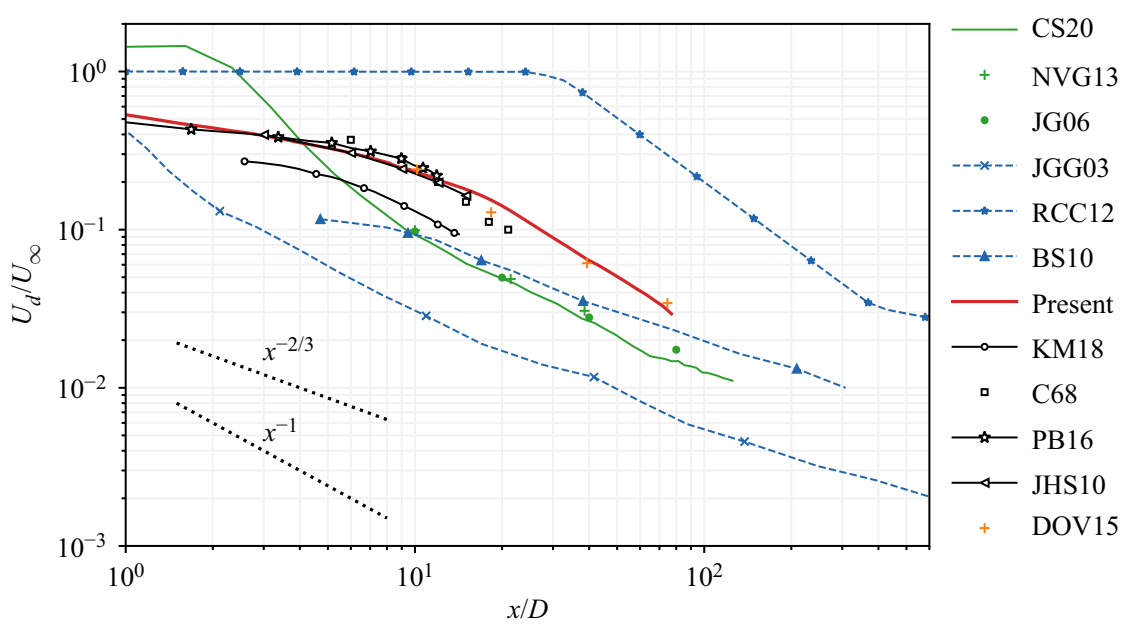

Figure 1. Decay of the defect velocity in various wake studies. Slender-body wakes (black): $\star$, black PB16, Posa \& Balaras (2016); $\square$, black C68, Chevray (1968); $\bigcirc$, black KM18, Kumar \& Mahesh (2018); $\triangleleft$, black JHS10, Jiménez, Hultmark \& Smits (2010). The red line is the present simulation. Disk wakes (green): -, green CS20, Chongsiripinyo \& Sarkar (2020); +, green NVG13, Nedić, Vassilicos \& Ganapathisubramani (2013); $\bigcirc$, green JG06, Johansson \& George (2006a). Temporal simulations (blue dashed lines): $\times$, blue JGG03, Johansson et al. (2003); $\star$, blue RCC12 case SD, Redford, Castro \& Coleman (2012); $\mathbf{\Delta}$, blue BS10, Brucker \& Sarkar (2010). Fractal plate (orange) +, orange DOV15, Dairay, Obligado \& Vassilicos (2015). The streamwise coordinate measures distance from the end of the wake generator.

Data from these studies are compiled in figure 1, which shows the decay of $U_{d}$ in, to the best of the authors' knowledge, all the available experiments and simulations of slender body wakes at high Reynolds numbers (in black). Slender-body wakes span up to $x / D \approx 20$ from the end of the body, a much shorter domain than studies with other wake generators which are also shown. The figure also includes recent studies of a disk (in green), temporally evolving wakes (in blue) and a fractal plate wake (in orange). Overall, the picture of wake decay remains unclear. The disk wakes show an initial decay similar to $U_{d} \sim x^{-1}$ and later on they transition to $U_{d} \sim x^{-2 / 3}$. The fractal plate decays following $U_{d} \sim x^{-1}$. The temporal simulations show significant variability owing to the use of different initialisation techniques and the transformation from $t$ to $x / D$. Some also show long initial regions where the decay is faster than the classic high-Re $U_{d} \sim x^{-2 / 3}$. All four of the slender-body wakes show a decay close to the classic $U_{d} \sim x^{-2 / 3}$. This result of $-2 / 3$ decay of $U_{d}$, which is derived by assuming full self-similarity of the flow, is somewhat puzzling since the length of the domain is limited in these studies and it is likely that only partial self-similarity is achieved.

The experimental evidence shows that the approach to self-similarity depends on the nature of the wake generator (Higuchi \& Kubota 1990). It also shows that universality, if ever achieved, is not achieved at distances relevant for practical applications (Wygnanski, Champagne \& Marasli 1986; Redford et al. 2012). All the available literature looking into the high- $R e$ far wake is devoted to bluff-body wakes. The present work is the first look into the far wake of a slender body at high Reynolds number. The main goal is to study the transition to self-similarity and ascertain if the far wake follows the classic high-Reynolds-number decay laws, as suggested by the previous body of literature. Unlike the wake of bluff bodies, the near wake of a slender body with a turbulent boundary layer is not dominated by vortex shedding (Jiménez et al. 2010; Kumar \& Mahesh 2018). In this work we also look into how this feature might affect the establishment and development 


\section{High-Reynolds-number wake of a slender body}

of the wake. Some of the questions that we address are as follows. (i) Does the decay of a slender-body wake at high Reynolds number conform to classical theory? (ii) How is the transition to self-similarity different from the wake of a bluff body? (iii) Why have some previous experiments and simulations shown wake exponents close to low- $R e$ values even at very high Reynolds numbers? (iv) Is there a possible relation between coherent structures and the wake decay?

To answer these questions, we simulate the wake of a $6: 1$ prolate spheroid with a tripped boundary layer. Unlike previous studies of slender-body wakes which reached up to a streamwise distance of $x / D=20$, here, we span $x / D=80$ at $R e=10^{5}$ to investigate the far wake. To the best of the authors' knowledge, this is the highest Reynolds number in a far-wake study that resolves the flow at a slender-body wake generator.

\section{Hybrid simulation}

To study the evolution of near-body flow into the far wake in a computationally feasible manner we employ a hybrid simulation. The hybrid simulation combines two simulations: a BI one that resolves the flow around the wake generator and a body-exclusive (BE) one that resolves the far wake. The BE simulation can be a temporally evolving wake (Pasquetti 2011) or a spatially evolving wake similarly to the approach of VanDine, Chongsiripinyo \& Sarkar (2018) who validated the method for a sphere wake. The use of the BI simulation avoids the drawback of regular temporal simulations, for which the choice of initial conditions introduces considerable variability in the subsequent wake evolution. In our implementation, the BI flow field provides the inflow for the subsequent BE simulation. The data from a selected cross-stream plane of the BI simulation are interpolated on to a new grid and fed into the BE simulation as an inlet boundary condition. This procedure allows us to relax the natural stiffness of the far-wake problem by using different spatial and temporal resolutions appropriate for the BI and BE stages. The grid cell in the BE simulation, which has to be sufficiently small to adequately resolve wake turbulence, is still much larger than that required to resolve the boundary layer. The consequently large time step in the BE simulation leads to considerable savings in computational time without compromising accuracy (VanDine et al. 2018).

The three-dimensional Navier-Stokes equations are solved in a cylindrical coordinate system using a staggered grid. An immersed boundary method (Balaras 2004; Yang \& Balaras 2006) is used to represent the $6: 1$ prolate spheroid. Both the BI and BE simulations are performed with the same solver. The solver uses a third-order Runge-Kutta method combined with second-order Crank-Nicolson scheme to advance the equations in time, and second-order-accurate central differences for spatial derivatives. This solver has been extensively validated in both unstratified and stratified sphere wakes (Pal et al. 2017). A wall-adapting local eddy-viscosity (WALE) model is used (Nicoud \& Ducros 1999). For both simulations, the boundary conditions are Dirichlet at the inflow, Orlanski for the convective outflow and Neumann at the lateral boundary. Further numerical details and validation are reported for a disk wake (Chongsiripinyo \& Sarkar 2020) and a lower-Re slender-body wake with laminar boundary layer (Ortiz-Tarin, Chongsiripinyo \& Sarkar 2019).

The cylindrical coordinate system is $(x, r, \phi)$ with the origin at the body centre. The minor-axis diameter of the prolate spheroid is $D$ and the major axis is aligned with the incoming velocity $U_{\infty}$. The body aspect ratio is $6: 1$ and the Reynolds number based on $D$ is $R e=U_{\infty} D / v=10^{5}$. To accelerate laminar-to-turbulent transition, the boundary layer is tripped by a small surface bump at $0.5 \mathrm{D}$ from the nose of the body. The bump has radial thickness of $0.002 D$ ( $\sim 15$ wall units) and streamwise extent of $0.1 D$. The location 


$\begin{array}{lcccccccc}\text { Case } & R e & L_{r} & L_{\phi} & L_{x}^{-} & L_{x}^{+} & N_{r} & N_{\phi} & N_{x} \\ \mathrm{BI} & 10^{5} & 5 & 2 \pi & -8 & 15 & 746 & 512 & 2560 \\ \mathrm{BE} & 10^{5} & 10 & 2 \pi & - & 80 & 479 & 256 & 4608\end{array}$

Table 1. Simulation parameters of the body-inclusive (BI) and body-exclusive (BE) simulations.
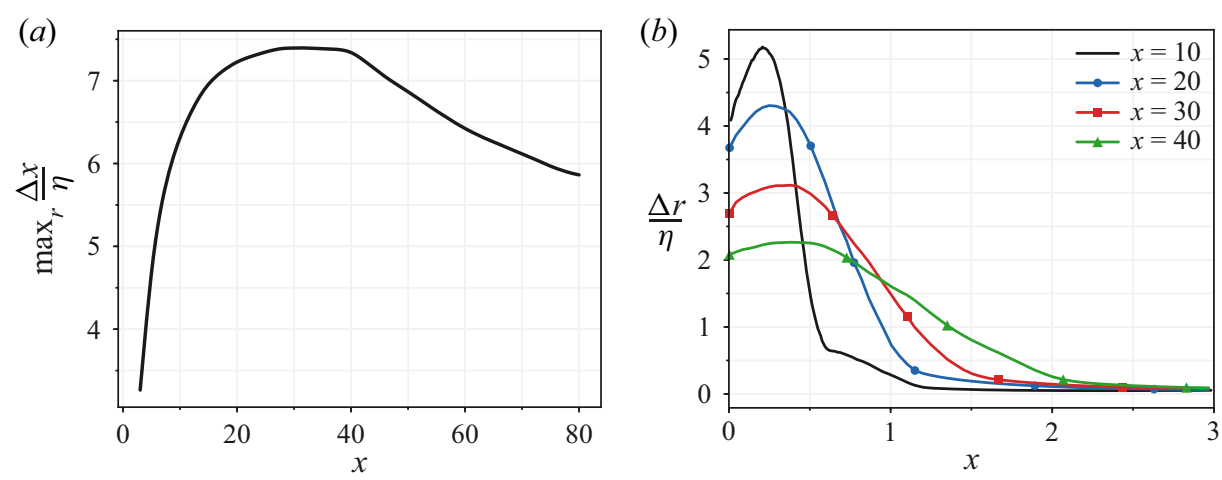

Figure 2. Grid quality: (a) radial maximum of the streamwise grid size over the Kolmogorov length scale; (b) radial grid size over the Kolmogorov length scale across the wake.

at which the flow time history from the BI simulation is extracted and subsequently fed as inflow into the $\mathrm{BE}$ simulation is $x / D=6$. This location is well away from the recirculation region which ends at $x / D=3.1$.

The simulation parameters are listed in table 1. The grid is designed to resolve the turbulent boundary layer and to resolve small-scale wake turbulence. The total number of grid points across BI and BE domains is approximately 1.5 billion. The turbulent boundary layer is resolved with $\Delta x^{+}=40, \Delta r^{+}=1$ and $r \Delta \phi^{+}=32$. There are 10 points in the viscous sublayer and 130 across the buffer and log layers. In the wake, the peak ratio between the grid size and the Kolmogorov length $\left(\eta=\left(v^{3} / \varepsilon\right)^{1 / 4}\right)$, in both $\mathrm{BI}$ and $\mathrm{BE}$ domains, is $\max (\Delta x / \eta)=7.5, \max (\Delta r / \eta)=6$ and $\max (r \Delta \theta / \eta)=5$. The distributions of $\Delta x / \eta$ and $\Delta r / \eta$ in the domain are given in figure 2. The peak ratio of the turbulent viscosity over the molecular viscosity occurs at the tripping location and is $v_{s g s} / v \approx 3$. Everywhere else, $v_{s g s} / v<1$, confirming the excellent resolution of the large-eddy simulation grid of both BI and BE simulations.

Flow statistics are obtained by temporal and azimuthal averaging. The average, denoted by \langle\rangle , is performed over $100 \mathrm{D} / U_{\infty}$ after the flow has reached statistically steady state. Velocity is normalised with the free-stream velocity $U_{\infty}$ and length with the body minor axis $D$. The mean velocities are $\left(U, U_{r}, U_{\phi}\right)$, the root-mean-square (r.m.s.) turbulent fluctuations $\left(u_{x}, u_{r}, u_{\phi}\right)$ and the Reynolds shear stress $u_{x r}$. The TKE is given by $k=\left(u_{x}^{2}+u_{r}^{2}+u_{\phi}^{2}\right) / 2$ and the turbulent dissipation rate by $\varepsilon=2 v\left\langle s_{i j} s_{i j}\right\rangle$ where $s_{i j}$ is the fluctuating strain-rate tensor. The decay rate $\alpha$ of any quantity is computed as the best fit of a power law, $f(x)=C\left(x-x_{0}\right)^{\alpha}$, to the data. The procedure employed here is similar to that of Obligado et al. (2016). The exponent is fitted first and then the amplitude and the virtual origin are obtained. Similarly to the experimental findings of Jiménez et al. (2010), keeping the virtual origin $x_{0}$ fixed had negligible effects on $\alpha$ or the fit quality. The $R^{2}$ regression value exceeds 0.98 in all cases. 


\section{High-Reynolds-number wake of a slender body}

(a)

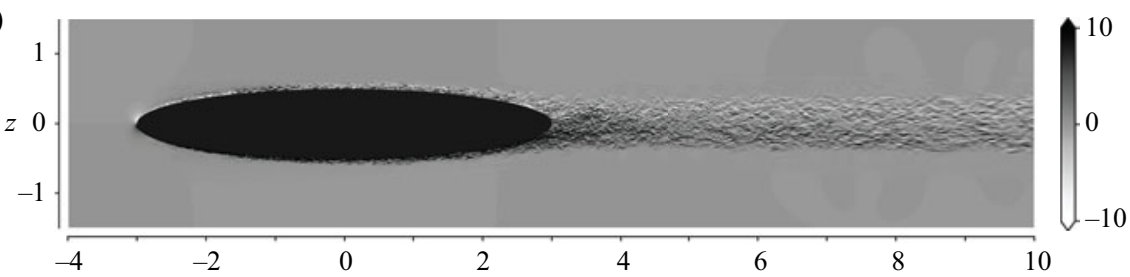

(b)
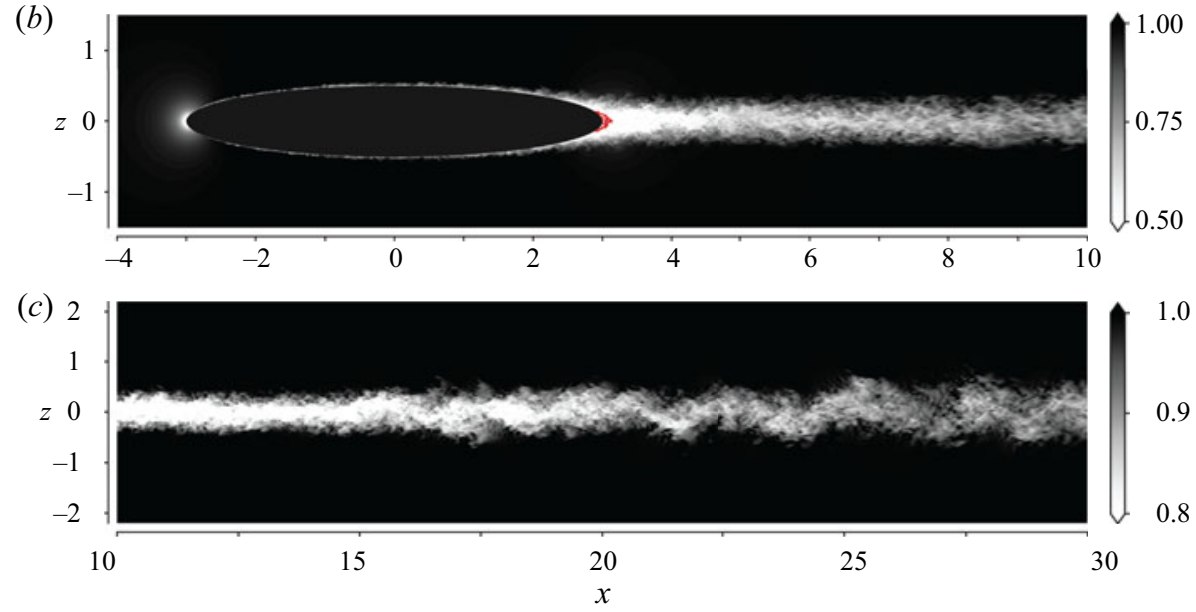

Figure 3. (a) Instantaneous lateral vorticity contour $\omega_{y}$ in the near wake, $(b)$ instantaneous streamwise velocity contour in the near wake and $(c)$ the intermediate wake. In $(b)$ the red isoline shows the limit of the recirculation region where the streamwise velocity is zero.

\section{Flow visualisation}

The visualisation of instantaneous vorticity and streamwise velocity in figure 3 reveals the main characteristics of the flow. The near wake is quasi-parallel and carries small-scale turbulence which has originated in the tripped boundary layer. The separation region, shown in red in figure $3(b)$, is small and extends for a distance of $0.1 D$ from the end of the body, in accordance with the experiments of Chevray (1968). The wake topology is similar to that observed in previous slender-body studies such as Kumar \& Mahesh (2018) and Posa \& Balaras (2016). Disk, plates and other bluff body wakes have significantly larger recirculation bubbles, e.g. the disk of Chongsiripinyo \& Sarkar (2020) has a recirculation bubble of length $2 D$. In addition, compared with the wake of bluff bodies, here the near wake is not dominated by the asymmetric shedding of the boundary layer, as was also observed by Kumar \& Mahesh (2018). In a bluff-body wake, the asymmetric shedding of the boundary-layer vorticity leads to the dominance of a helical mode immediately after the recirculation bubble, e.g.Berger, Scholz \& Schumm (1990), Johansson \& George (2006b) and Nidhan et al. (2020). Figure 3(a,b) shows a quasi-cylindrical structure in the near wake. Only farther downstream beyond $x \approx 20$, (figure $3 c$ ), does the wake start showing a large-scale sinuous structure.

\section{The classic axisymmetric wake theory}

The classic analysis of the axisymmetric turbulent wake starts with the Reynolds-averaged streamwise momentum equation,

$$
U \frac{\partial U}{\partial x}+U_{r} \frac{\partial U}{\partial r}=-\frac{1}{r} \frac{\partial}{\partial r}\left(r u_{x r}\right)-\frac{1}{\rho} \frac{\partial p}{\partial x} .
$$


By writing the left-hand side as a function of the momentum deficit $U_{\infty}-U$, assuming that the momentum deficit is $U_{\infty}-U \ll U_{\infty}$ and retaining only the first-order terms, one obtains

$$
U_{\infty} \frac{\partial\left(U-U_{\infty}\right)}{\partial x}=-\frac{1}{r} \frac{\partial}{\partial r}\left(r u_{r x}\right)
$$

where we have used the boundary-layer approximation and assumed high $R e$ so that the viscous term is negligible.

The analysis proceeds by assuming self-similarity of the velocity deficit and the Reynolds stresses,

$$
U_{\infty}-U=U_{d}(x) f(\eta), \quad u_{r x}=R_{s}(x) g(\eta),
$$

where $\eta=r / L$ is a similarity variable that depends on a characteristic wake width $L=$ $L(x)$. Here $U_{d}$ and $R_{s}$ are the peak values of the velocity deficit and the Reynolds shear stress, respectively. Equation (4.2) then simplifies to

$$
\left[\frac{L}{U_{d}} \frac{\mathrm{d} U_{d}}{\mathrm{~d} x}\right] f-\left[\frac{\mathrm{d} L}{\mathrm{~d} x}\right] \eta f^{\prime}=\left[\frac{R_{s}}{U_{\infty} U_{d}}\right] \frac{1}{\eta} \frac{\mathrm{d}}{\mathrm{d} \eta}(\eta g),
$$

where, for self-preservation, all the terms in brackets must have the same $x$ dependence.

The same procedure applied to the conservation of TKE leads to

$$
\left[\frac{L}{K_{s}} \frac{\mathrm{d} K_{s}}{\mathrm{~d} x}\right] h-\left[\frac{\mathrm{d} L}{\mathrm{~d} x}\right] \eta h^{\prime}=-\left[\frac{D_{s} L}{K_{s} U_{\infty}}\right] e+\left[\frac{T_{s} L}{K_{s} U_{\infty}}\right] t+\left[\frac{P_{s} L}{K_{s} U_{\infty}}\right] p,
$$

where the TKE, turbulent dissipation, production and transport terms are as follows,

$$
k=K_{s}(x) h(\eta), \quad \varepsilon=D_{s}(x) e(\eta), \quad P=P_{s}(x) p(\eta) \quad \text { and } \quad T=T_{s}(x) t(\eta) . \quad(4.6 a-d)
$$

As in (4.4), for full self-similarity, all the bracketed terms in (4.5) must have the same $x$ dependence. However, flows can be partially self-preserving, meaning that they show self-similarity only in the mean momentum equation or only up to certain orders of the turbulence statistics (George 1989).

The integration of the momentum equation over a cross-section provides us with an additional constraint, namely, the momentum thickness $\theta(x)$, or its first-order approximation $\tilde{\theta}(x)$, is constant. Here,

$$
\theta^{2}=\frac{1}{U_{\infty}^{2}} \int_{0}^{\infty} U\left(U_{\infty}-U\right) r \mathrm{~d} r \quad \text { and } \quad \tilde{\theta}^{2}=\frac{1}{U_{\infty}} \int_{0}^{\infty}\left(U_{\infty}-U\right) r \mathrm{~d} r
$$

and $\theta \approx \tilde{\theta}$ holds only when $U_{d} / U_{\infty} \ll 1$. After employing $(4.3 a, b)$ for the momentum deficit, the expression for $\tilde{\theta}$ can be readily integrated to obtain $L^{2} U_{d}=\tilde{\theta}^{2} U_{\infty}$, which directly relates $L$ and $U_{d}$.

At this point, to obtain the decay law of $U_{d}$, more assumptions are required and classic theories start to differ. Tennekes \& Lumley (1972) assume that $R_{S} \sim U_{d}^{2}$ and, using (4.4), they obtain $U_{d} \sim x^{-2 / 3}$. Here $R_{s}$ can vary independently of $U_{d}$ and then the TKE equation comes into play. Townsend (1976) and George (1989) assume that dissipation follows the high-Re inertial scaling $\varepsilon \sim K^{3 / 2} / L$ and that the turbulent production is $P=-u_{x r}(\partial U / \partial r) \sim R_{s}\left(U_{d} / L\right)$. With these assumptions, they showed that $K_{s} \sim R_{s} \sim U_{d}^{2}$ and also $U_{d} \sim x^{-2 / 3}$. In addition, George (1989) obtained the low-Re $U_{d} \sim x^{-1}$ decay when the viscous term $\sim v U_{d} / L^{2}$ dominates the turbulent stress in the momentum balance and turbulent dissipation scales as $\varepsilon \sim \nu U_{d}^{2} / L^{2}$. 


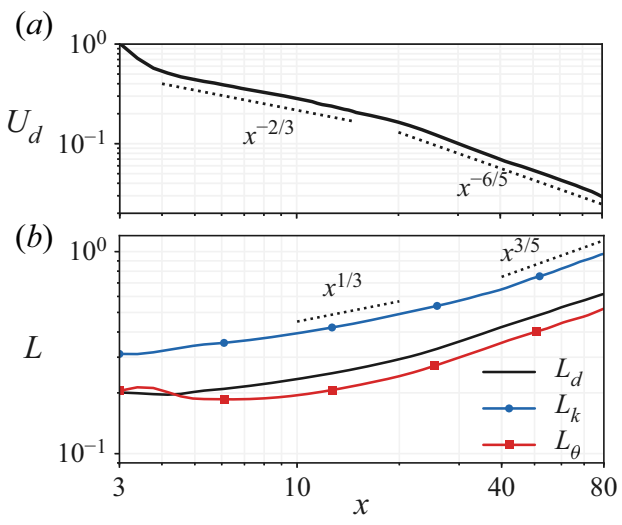

(c)

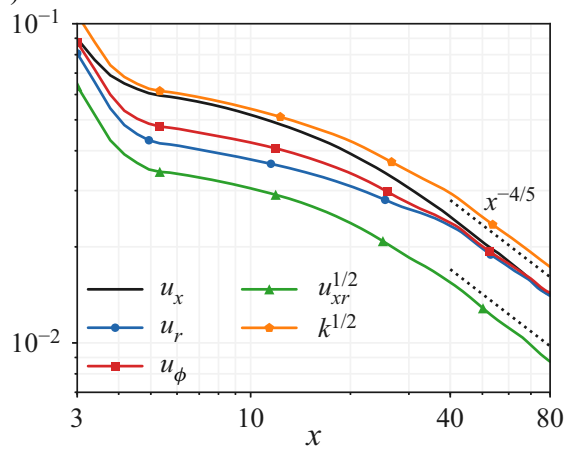

Figure 4. Streamwise evolution of wake statistics: $(a)$ centreline defect velocity, $(b)$ wake half-widths and (c) peak r.m.s. velocities, Reynolds shear stress and TKE.

\section{Wake analysis}

In the present study, the decay rates of near-wake statistics $(x<20)$ agree well with previous experiments and simulations of a slender-body wake. However, the behaviour after $x=20$ has not been documented before. We find that after $x=20$, the flow transitions from partial to full self-similarity, while showing notable departures from the classic wake decay.

Figure 4 shows the streamwise evolution of several wake statistics. The peak defect velocity $U_{d}$ shows two distinct decay rates. The near wake, $5<x<15$, decays in a similar manner to the classic high-Re result, $U_{d} \sim x^{-0.66}$. However, in the intermediate and far wake, $20<x<80$, the decay rate increases to $U_{d} \sim x^{-1.2}$. Figure $4(b)$ shows the evolution of the wake width $(L)$ measured in three different ways. $L_{d}$ is defined such that $U\left(L_{d}\right)=U_{d} / 2$, and $L_{k}$ is defined analogously using the TKE as $k\left(L_{k}\right)=k(r=0) / 2$. The displacement thickness $\left(L_{\theta}\right)$ is defined by $L_{\theta}^{2}=U_{d}^{-1} \int_{0}^{\infty}\left(U_{\infty}-U\right) r \mathrm{~d} r$. For $x>6$, the three measures of $L$ show the same values of growth rate. The wake width grows as $L \sim x^{0.33}$ in the region $15<x<20$ and transitions towards $L \sim x^{0.6}$ as the wake traverses $30<x<80$.

Figure 4(c) shows the decay of the peak r.m.s. turbulent intensities together with the TKE and the Reynolds shear stress. The near wake has anisotropy with $u_{x}>u_{\phi}>u_{r}$ and the individual decay rates agree well with Jiménez et al. (2010). In particular, $u_{r}, u_{\phi} \sim$ $x^{-0.21}$ and $u_{x}, u_{x r}^{1 / 2} \sim x^{-0.26}$. After $x \approx 40$, the r.m.s. values become similar and they show a long region of constant decay rate. Here, the TKE and the Reynolds shear stress decay as $k^{1 / 2}, u_{x r}^{1 / 2} \sim x^{-0.8}$ and the r.m.s. velocities as $u_{r}, u_{\theta} \sim x^{-0.73}$ and $u_{x} \sim x^{-0.86}$.

To proceed with the classic wake analysis, we move on to assess self-similarity. Figure 5 shows the profiles of velocity deficit, TKE, Reynolds shear stress $\left(u_{x r}\right)$ and dissipation rate $(\varepsilon)$, each scaled by its peak, as a function of normalised radial coordinate at different streamwise locations.

The $U$-deficit profile exhibits self-similarity from $x \approx 5$ onwards. The $u_{x r}$ profiles collapse together in local scales after $x \approx 10$. TKE shows self-similarity only after $x \approx 30$ and $\varepsilon$ after $x \approx 40$. In the near wake, $k$ and $\varepsilon$ show an off-centre maximum, which is the imprint of the tripped boundary layer. Indeed, self-similarity of higher-order statistics require longer $x$, thus revealing stages of partial self-similarity (George 1989). Terms in 

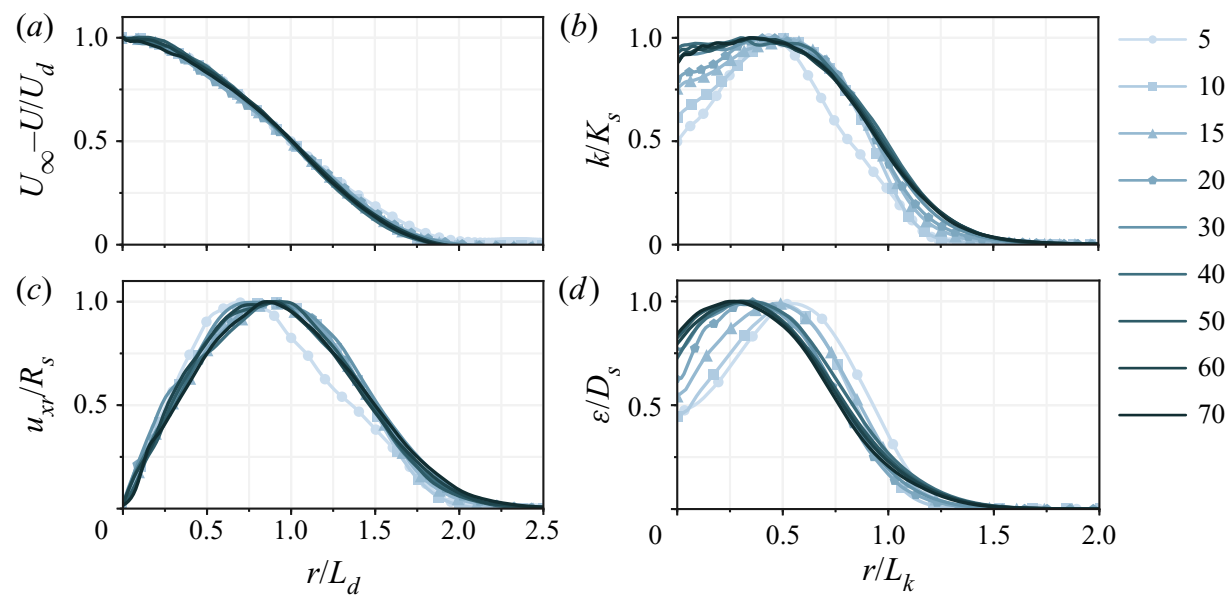

Figure 5. Radial profiles in similarity coordinates at various streamwise locations: $(a)$ defect velocity, (b) TKE, $(c)$ turbulent shear stress and $(d)$ turbulent dissipation.
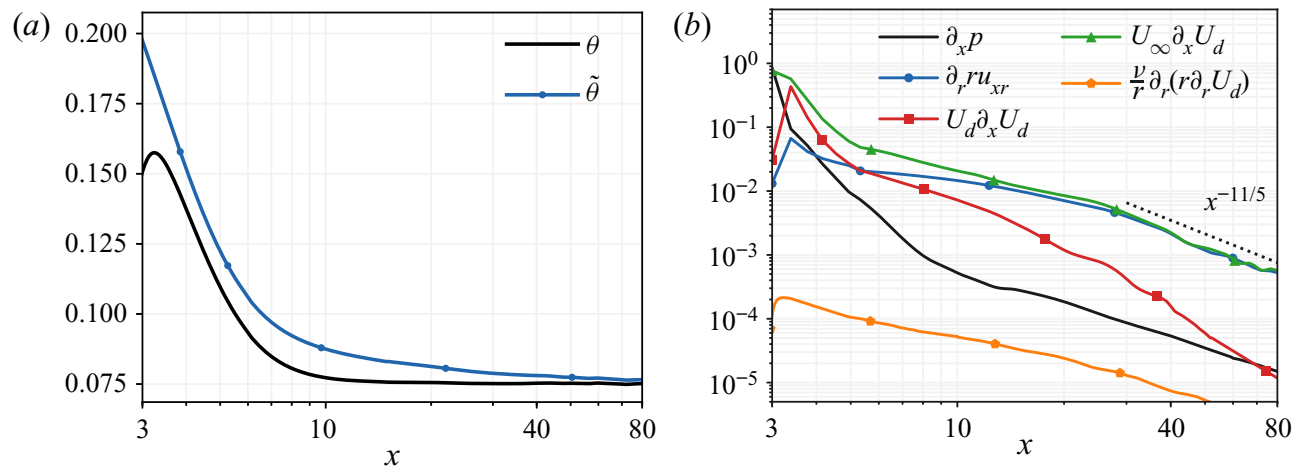

Figure 6. (a) Streamwise evolution of the momentum thickness $\theta$ and the simplified momentum thickness $\tilde{\theta}$. (b) Streamwise evolution of terms in the streamwise momentum balance (4.1). The convective term has been written using $U_{d}=U_{\infty}-U$. The viscous term is also plotted.

the momentum equation show self-similar behaviour after $x \approx 10$ and terms in the TKE equation after $x \approx 40$.

In addition to the assumption of self-similarity, the classic turbulent wake theory assumes that the defect velocity $U_{d}$ is small compared with the free-stream velocity $U_{\infty}$. This hypothesis simplifies the equations significantly, leads to the classic scaling laws, and to the integral constraint $U_{d} L^{2}$ being constant at any cross-section along the wake. Figure 6(a) shows the evolution of the momentum thickness $\theta$ and the first-order approximation of the momentum thickness $\tilde{\theta}$. The full momentum thickness reaches a constant value of $\theta_{0}=0.075$ after $x=10$. The simplified momentum thickness does not become constant until $x \approx 60$ and in the near wake it changes significantly. This explains why the growth rate of $L$ and the decay of $U_{d}$ do not fulfil exactly $U_{d} L^{2}=U_{\infty} \theta_{0}^{2}$ in the near wake. Note that, in the near-wake region $5 \leq x \leq 15$, the simulation shows $L \sim x^{0.26}$ and $U_{d} \sim x^{-0.66}$, i.e. $U_{d} L^{2}$ is not constant.

To further understand the approach to similarity, the centreline evolution of the leading terms in the $U$ equation are evaluated and plotted in figure $6(b)$. The second-order 


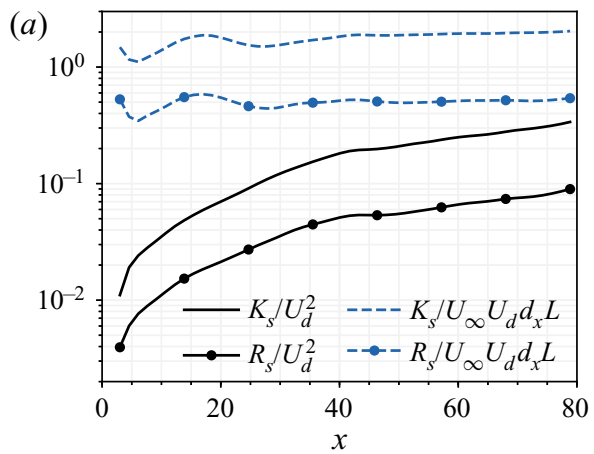

(b)

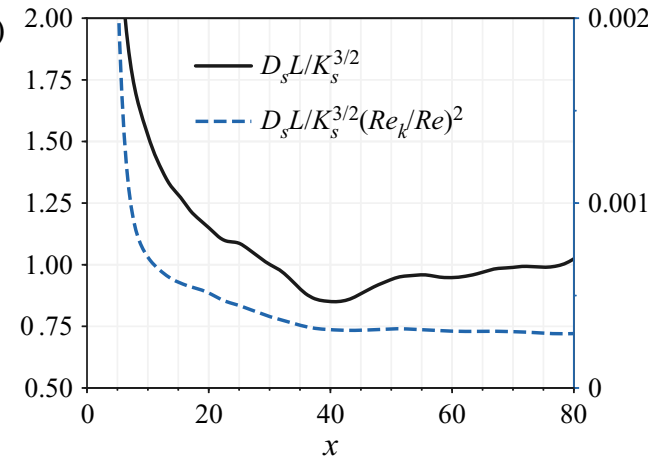

Figure 7. Evaluation of alternative scalings. (a) Peak TKE and shear stress scaled with the classic scaling $\left(U_{d}^{2}\right)$ and with $U_{\infty} U_{d} d_{x} L$. (b) Peak turbulent dissipation normalised with classical and with non-equilibrium estimates.

term, $U_{d}\left(\partial U_{d} / \partial x\right)$, remains non-negligible until $x \approx 15$. Equation (4.2) becomes a better approximation of the exact $U$-equation with increasing $x$ but complete balance between the decay of $U$ and the radial derivative of $u_{x r}$ is not achieved until $x \approx 25$. Note that the viscous term remains at least two orders of magnitude below the leading terms. At comparable $R e$, blunt-body wakes show a higher initial value of $U_{d}$ however, because their entrainment levels are higher, $U_{d}$ decays faster. Therefore, in the range $x \approx 10-20, U_{d}$ of a blunt-body disk wake becomes half that of the spheroid wake as is evident after comparing the black and the green lines in figure 1. At $x=10$ the disk of Chongsiripinyo \& Sarkar (2020) exhibits a value of $U_{d} / U_{\infty}=0.09$ and at $x=30$ the value is $U_{d} / U_{\infty}=0.03$. Here, at $x=10$ the magnitude is $U_{d} / U_{\infty}=0.22$ and, at $x=30, U_{d} / U_{\infty}=0.06$. This factor-of-two-larger value of $U_{d}$ for the prolate spheroid relative to the sphere wake at comparable $x$ implies that a longer streamwise distance is required in the spheroid wake for the second-order terms to truly become negligible.

The first-order approximation of the momentum conservation (4.2) and the first-order approximation of the momentum thickness definition $(4.7 a, b)$ have been used to explain the observed decay of $U_{d}$ in the near wake of slender bodies since the classic wake theories were established (Chevray 1968; Jiménez et al. 2010; Kumar \& Mahesh 2018). They both rely on the assumption that $U_{d} \ll U_{\infty}$ which, based on the present results, is not a good approximation in the near wake of a streamlined body.

After testing the validity of the assumptions involving the velocity deficit and the transverse Reynolds stress, we now move to the scaling of TKE and turbulent dissipation $\varepsilon$. Figure $7(a)$ shows the peak TKE $\left(K_{s}\right)$ and the peak turbulent shear stress $\left(R_{s}\right)$ scaled by $U_{d}^{2}$. Neither becomes constant, which implies that, at least until $x=80, U_{d}^{2}$ is not the proper scaling for $k$ and $u_{x r}$. Instead, they satisfy $K_{s} \sim R_{s} \sim U_{\infty} U_{d}(\mathrm{~d} L / \mathrm{d} x)$. The r.m.s. velocities $u_{r}, u_{\phi}, u_{x}$ also show the same trend individually. This scaling was observed in the wake of a fractal plate by Dairay et al. (2015) and it can be obtained from (4.4), introducing $K_{S} \sim R_{s}$ as observed in the simulation.

The inertial estimate, $\varepsilon \sim K_{S}^{3 / 2} / L$, is tested for the normalised peak dissipation $\left(D_{S}\right)$, black solid curve in figure 7(b). This curve does not asymptote to a constant, showing that inertial scaling does not hold in the region $x<80$. An alternative is the non-equilibrium scaling proposed by Nedić et al. (2013),

$$
\varepsilon=\left(\frac{R e}{R e_{k}}\right)^{n} \frac{k^{3 / 2}}{L}
$$


where $R e_{k}=\sqrt{k} L / v$ is the local Reynolds number. The non-equilibrium estimate, specifically with $n=2$ in (5.1), is found to be a much better choice to scale $D_{s}$ beyond $x=40$ as shown by the dashed curve in figure $7(b)$. The non-equilibrium scaling of dissipation has been observed in bluff-body wakes (Nedić et al. 2013; Dairay et al. 2015; Obligado et al. 2016; Chongsiripinyo \& Sarkar 2020) and jets (Cafiero \& Vassilicos 2019). It has also been found to hold in unsteady decaying turbulence (Goto \& Vassilicos 2016), where it was shown to be related to the imbalance between the large-scale energy of the turbulence and the small-scale dissipation. Whereas in previous bluff-body studies $n=1$ was the appropriate exponent to scale dissipation, here $n=2$ is a better choice. The different exponent might be related to a different mechanism sustaining the large-scale wake motions, here the imprint of vortex shedding from the body is weak, large-scale coherent structures are only observed late in the wake, and the TKE and dissipation profiles show an off-centre peak whereas in bluff-body wakes such as those studied by Dairay et al. (2015) and Nidhan et al. (2020), the maxima occur at the centreline. Further investigation is still required for a more comprehensive understanding of the non-equilibrium scaling (Vassilicos 2015; Cafiero \& Vassilicos 2019). In addition, assessment of whether the turbulent dissipation transitions to the classic inertial estimate at larger downstream distances will require longer spatial domains.

At this point, we have enough information to obtain power laws for $\left\{U_{d}, L, K_{s}, R_{s}, D_{s}\right\}$ in the self-similar far wake. Let $U_{d} \sim x^{-\alpha}$. Then $L \sim x^{\alpha / 2}$ to satisfy the momentum integral constraint. Let $K_{s} \sim x^{-\beta}$. Equation (5.1) with $n=2$ leads to $D_{s} \sim x^{-(\beta+3 \alpha) / 2}$. We have shown previously that $K_{s} \sim R_{s} \sim U_{\infty} U_{d}(\mathrm{~d} L / \mathrm{d} x)$, so $\beta=1+\alpha / 2$. After balancing the TKE decay with the dissipation, equation (4.5) provides us with an additional constraint for $\alpha$, whose solution is $\alpha=6 / 5$. Finally,

$$
U_{d} \sim x^{-6 / 5}, \quad L \sim x^{3 / 5}, \quad K_{s} \sim R_{S} \sim x^{-8 / 5} \quad \text { and } \quad D_{s} \sim x^{-13 / 5},
$$

which agree well with the growth rates observed in the simulation for the region $40<x<$ 80 ,

$$
U_{d} \sim x^{-1.20}, \quad L \sim x^{0.59}, \quad K_{s} \sim x^{-1.60} \quad R_{s} \sim x^{-1.64} \quad \text { and } \quad D_{s} \sim x^{-2.63} .
$$

It is worth noting that the production, which scales as $P_{s} \sim x^{-17 / 5}$, drops from the leading-order TKE balance.

Here, it is necessary to remark on the near-wake behaviour. The observed power laws over $5<x<15$ are $U_{d} \sim x^{-2 / 3}$ and $L \sim x^{0.26}$. Our simulation and all the previous evidence (see figure 1 ) show a region of $U_{d} \sim x^{-0.66}$ over $5 \lesssim x \lesssim 15$. However, in this region, the profiles of $u_{x r}, k$ and $\varepsilon$ are not self-similar. Thus, (4.4) and (4.5) cannot be invoked. Furthermore, $R_{s} \nsim U_{d}^{2}$. The observed $U_{d} \sim x^{-0.66}$ is probably a short-lived coincidence and not the asymptotic $-2 / 3$ decay. In this region, the first-order momentum integral constraint $\left(U_{d} L^{2}\right)$ is not constant and, hence, $L \nsim x^{0.33}$. Instead, $L$ grows at a slower rate, $L \sim x^{0.26}$.

There are three potential causes for the change of wake power laws from the near to far wake: (i) after a transient, the mean flow and turbulence achieve equilibrium, (ii) the local Reynolds number reduces significantly to bring viscosity into play, or (iii) there is a structural change in the wake. In figure 7 , it was shown that turbulence and mean flow are not in equilibrium since $k \nsim U_{d}^{2}, R_{s} \nsim U_{d}^{2}$ throughout the domain. Regarding (ii), the flow does not show a transition to low local Reynolds number. As can be seen in figure 8, both $R e_{L}=U_{d} L / v$ and $R e_{k}$ are of the order of $10^{3}$. In their simulations, Johansson et al. (2003) observed that the flow transitioned to low-Re decay once the local Reynolds number went below 500, here the local Reynolds numbers are well above that threshold. In addition, the 


\section{High-Reynolds-number wake of a slender body}

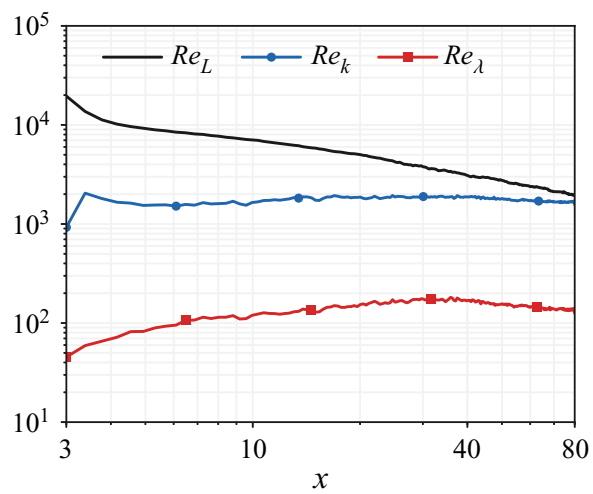

Figure 8. Streamwise evolution at the centreline of different local Reynolds numbers: $R e_{L}=U_{d} L_{d} / \nu$, $R e_{k}=\sqrt{k} L_{k} / v$ and $R e_{\lambda}=\sqrt{k} \lambda / v$, where $\lambda$ is the Taylor microscale.

(a)

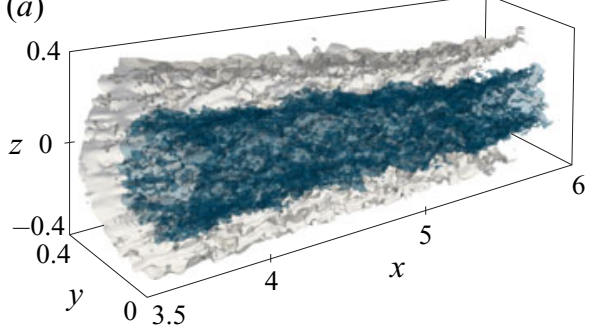

(b)

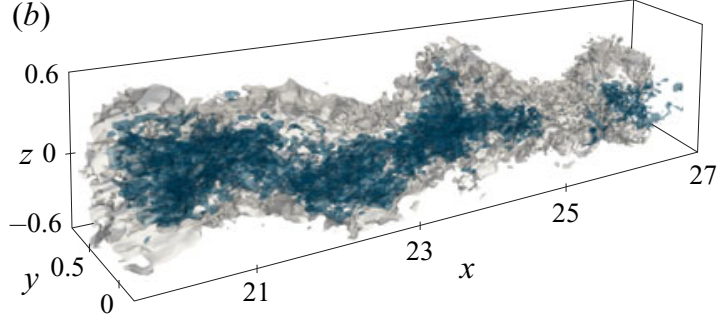

Figure 9. Isocountours of instantaneous streamwise velocity: (a) the near wake, white $0.97 U_{\infty}$, blue $0.7 U_{\infty}$; and $(b)$ the intermediate wake, white $0.97 U_{\infty}$, blue $0.85 U_{\infty}$.

microscale Reynolds number, $R e_{\lambda}=\sqrt{k} \lambda / v$ where $\lambda^{2}=15 v u_{x}^{2} / \varepsilon$, is above 100 and the energy spectra show a $-5 / 3$ decay throughout the domain (figure $10 b$ ).

Regarding (iii), the emergence or decay of coherent structures is a major structural change. The three-dimensional visualisation of figure 9 shows the emergence of a helical structure coinciding with the $U_{d} \sim x^{-2 / 3}$ to $U_{d} \sim x^{-6 / 5}$ transition. The near wake (figure $9(a)$, also evident in figure $3 a, b)$ is quasi-parallel without any visible large-scale azimuthal asymmetry. This near-wake topology is similar to that found by Kumar \& Mahesh (2018) and Posa \& Balaras (2016). However, farther downstream (figure 9b) a sinuous single helix, originating at $x \approx 20$, is observed. Previous simulations and experiments did not access locations downstream of $x \approx 20$ and, hence, did not observe the emergence of helical structures in the intermediate wake region.

To further characterise the sinuous mode, figure 10(a) shows the cross-sectional area-integrated energy of the streamwise velocity fluctuations as a function of the azimuthal mode $(m)$. Initially, at $x=3.5$, the turbulence is nearly broadband. As the wake evolves, energy concentrates around the $|m|=1$ mode and, progressively, the single helical mode dominates. The spectra (figure 10b) of $u_{x}$ confirms this finding. After $x=10$, a peak at $S t=f D / U=0.28$ is visible and is sustained until the end of the domain. $S t=0.28$ corresponds to the wavelength of the undulation identified in figure $9(b)$.

We can further investigate the structure of the wake by looking into the spatio-temporal evolution of the azimuthal modes. Figure $11(a, c, e, g)$ show the time evolution of the Fourier 

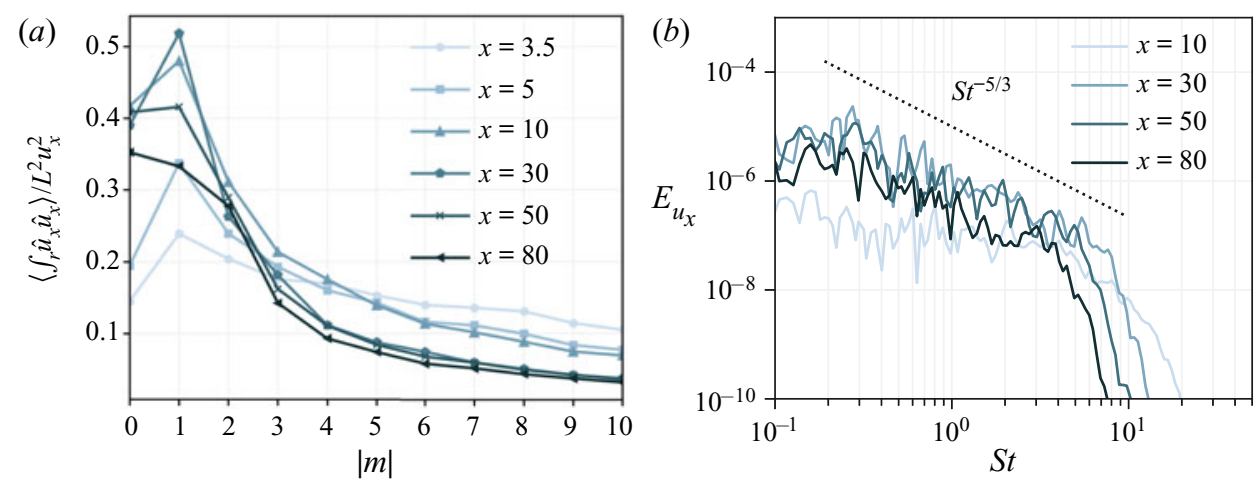

Figure 10. (a) Cross-wake area-integrated energy of $u_{x}$ as a function of the azimuthal wavenumber $|m|$. (b) Energy spectra of $u_{x}$ at $r=0.5$.

coefficients of the axisymmetric $m=0$ mode and the single helix $|m|=1$ mode. The $r-t$ variation of these modes is plotted at two streamwise locations: $x=3.5$ and $x=$ 30 , representative of the near and the intermediate wake, respectively. The characteristic frequencies of these modes are also identified by performing a temporal Fourier transform. The corresponding spectra are shown in figure $11(b, d, f, h)$.

In the near wake at $x=3.5$, which is $0.5 D$ from the trailing end of the spheroid, the $|m|=0$ axisymmetric mode is broadband and carries the small-scale turbulence of the boundary layer, see figure $11(a, b)$. The $|m|=1$ mode (figure 11c), however, has distinctive low-frequency motions in the wake core. This low frequency is caused by the oscillation of the recirculation region, which is small and spans $r \approx 0.1$ as was shown in figure 3(b). In the spectra of figure $11(d)$, two low-frequency peaks are observed: $S t=0.16$ and $S t=$ 0.28 . At $x=3.5$, both $m=0$ and $|m|=1$ have energy levels comparable with the other nearby modes, as was shown in figure $10(a)$.

At $x=30$, the small scales of the boundary layer have been dissipated and the $|m|=1$ low-frequency motion spans the whole wake width. A peak with a characteristic frequency of $S t=0.28$ is clearly identified in figure $11(h)$ and is visible in the periodic stripes of figure $11(\mathrm{~g})$. This peak is also observed farther downstream, as was shown in figure $10(\mathrm{~b})$ and corresponds to the undulation observed in the wake visualisations, figures $3(c)$ and $9(b)$. In this region, the asymmetric $|m|=1$ mode contributes the most to the energy of the flow. Note that the origin of the $S t=0.28$ oscillation can be traced to the near wake. However, its effect as a coherent structure is not visible until $x \approx 20$.

It is well established by experiments and linear stability analysis (Sato \& Okada 1966; Wygnanski et al. 1986; Monkewitz 1988; Berger et al. 1990; Rigas et al. 2014), that the wake of axisymmetric bodies is dominated by a global instability with azimuthal wave number $|m|=1$. In bluff-body wakes, the $|m|=1$ mode dominates the flow starting right at the recirculation region where there is strong asymmetric shedding of the boundary layer (Rigas et al. 2014; Nidhan et al. 2020). In this wake, however, the recirculation region is much smaller, and the asymmetry induced by the separated flow is weaker. The asymmetry is particularly weak because the flow immediately behind the body is surrounded by an axisymmetric region of turbulence originating from the turbulent boundary layer. As a result, the large-scale symmetry breaking of the wake associated with the presence of a coherent helical structure, becomes dominant only farther downstream, at $x \approx 20$, when the imprint of the boundary-layer turbulence becomes sufficiently weak. 

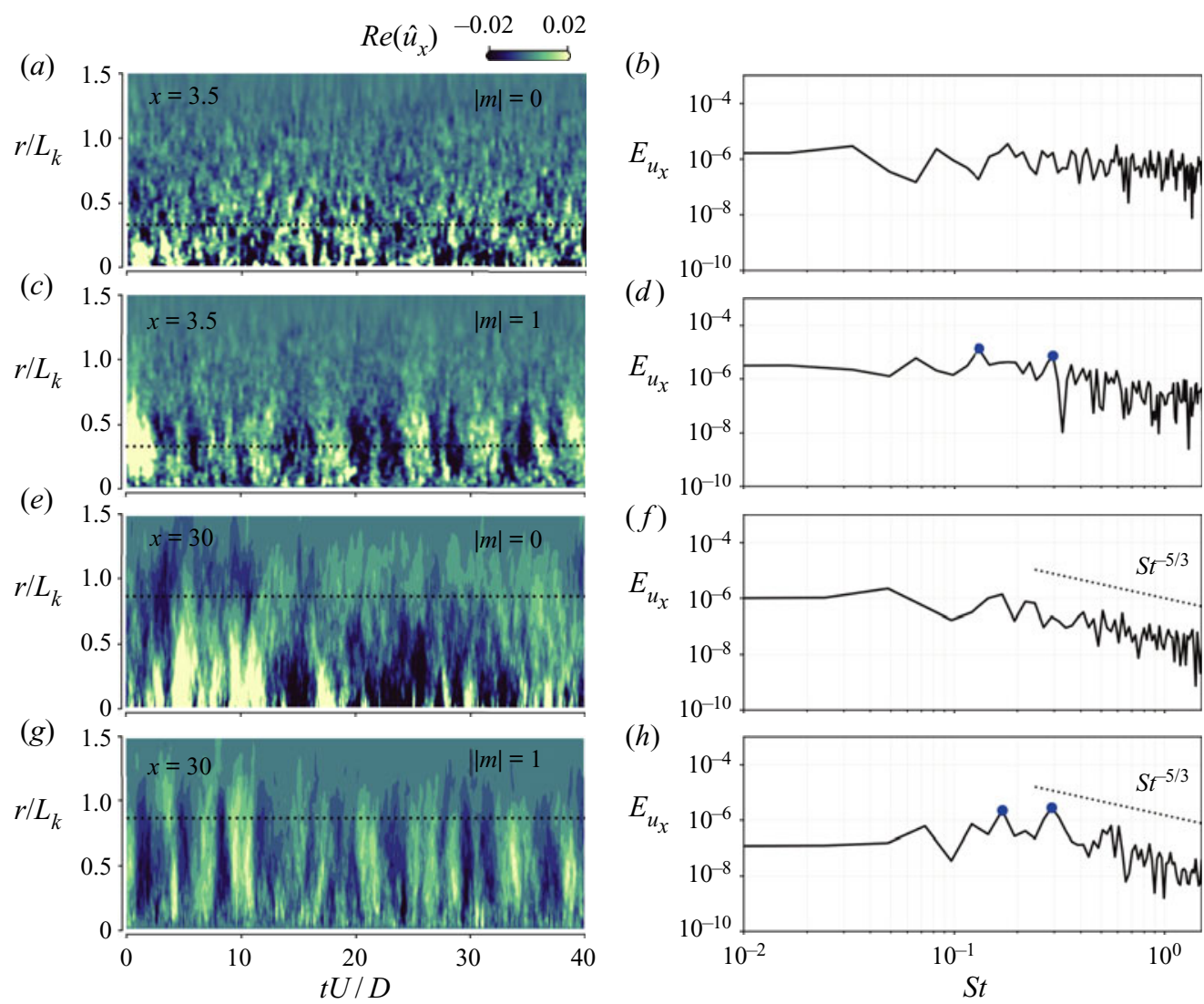

Figure 11. Azimuthal Fourier coefficients of modes $|m|=0$ and $|m|=1$ as a function of the radial coordinate and time at a fixed streamwise location: $(a, c)$ near wake at $x=3.5$, which is $0.5 D$ downstream from the trailing end of the body; $(e, g)$ intermediate wake at $x=30$. Corresponding power spectra of the modes computed at specific radial locations, marked by the dotted black line: $(b, d)$ near-wake spectra computed at $r=0.1 ;(f, h)$ intermediate-wake spectra at $r=0.5$.

The dominance of the $|m|=1$ mode in the intermediate wake coincides with the change of the decay rate and precedes the non-equilibrium scaling of dissipation. The relation between the non-equilibrium scaling of dissipation and the presence of coherent structures was hypothesised by Goto \& Vassilicos (2016) in three-dimensional decaying turbulence and more recently by Cafiero \& Vassilicos (2019) in turbulent jets. Here also, there is evidence of such a connection. In previous bluff-body wake studies (Chongsiripinyo \& Sarkar 2020; Nidhan et al. 2020), the dominance of the helical $|m|=1$ instability and the establishment of a region of non-equilibrium scaling occurs very early in the wake. In the slender-body wake, the helical mode develops farther downstream and so does the non-equilibrium region. Note the strong contribution to the energy of the $|m|=1$ mode at $x=30$ in figure $10(a)$ immediately before the non-equilibrium scaling becomes appropriate.

Farther downstream, near the end of the domain, as shown in figure $10(a)$, the dominance of $|m|=1$ recedes. Therefore, the far wake might eventually transition to the classic $U_{d} \sim x^{-2 / 3}$. Simulations or experiments with longer domains are needed to clarify this. 


\section{Conclusions}

The turbulent wake of a slender body has been studied using a high-resolution hybrid simulation. The wake generator is a $6: 1$ prolate spheroid with a tripped boundary layer and the diameter-based Reynolds number is $R e=10^{5}$. To the best of the authors' knowledge this is the highest $R e$ considered in a far-wake study that solves the flow around the wake generator.

Previous investigations of slender-body wakes with turbulent boundary layers have reached a maximum downstream distance that does not exceed $x / D=18$ (Chevray 1968; Jiménez et al. 2010; Posa \& Balaras 2016; Kumar \& Mahesh 2018). Until $x / D=18$, the wake decay rate is similar to the classic high-Re behaviour: $U_{d} \sim x^{-2 / 3}$. However, in this study the domain extends until $x / D=80$ and we show that, after $x / D \approx 20$, the decay rate changes to $U_{d} \sim x^{-6 / 5}$.

In the near-wake region $(x / D \lesssim 20)$, the classic self-similar turbulence scalings $(k \sim$ $u_{x r} \sim U_{d}^{2}$ and $\left.\varepsilon \sim k^{3 / 2} / L\right)$ that lead to the asymptotic $U_{d} \sim x^{-2 / 3}$ decay do not hold. The variables have not reached full self-similarity, the magnitude of the second-order terms is still significant and the observed $U_{d} \sim x^{-2 / 3}$ is probably a short-lived coincidence with the classic wake result. After $x / D=40$, profiles of $k$ and $\varepsilon$ exhibit self-similarity. The transition to the observed far-wake decay law of $U_{d} \sim x^{-6 / 5}$ is derived from the observed non-equilibrium scaling of dissipation $\varepsilon \sim\left(\operatorname{Re} / \operatorname{Re}_{k}\right)^{2} k^{3 / 2} / L$, which was used previously to explain non-classical decay rates in bluff-body wakes (Nedić et al. 2013; Dairay et al. 2015; Obligado et al. 2016; Chongsiripinyo \& Sarkar 2020).

The change in wake decay cannot be attributed to the low-Re asymptotic result $U_{d} \sim$ $x^{-1}$, because the local wake Reynolds numbers, $R e=U_{d} L / v, R e_{k}=\sqrt{k} L / v$ and $R e_{\lambda}=$ $\sqrt{k} \lambda / \nu$, where $\lambda$ is the Taylor microscale, remain large throughout the domain. The decay rate transitions in the region where there is a structural change in the wake, dominance of a sinuous single helix $(|m|=1)$ azimuthal mode. In a bluff-body wake the strong asymmetric shedding of the boundary layer leads to a dominant helical mode immediately after the recirculation bubble (Berger et al. 1990; Johansson \& George 2006b; Nidhan et al. 2020). Here, the separated region behind the slender-body is very small (approximately $0.1 D$ ), the near wake is quasi-parallel and dominated by the boundary-layer turbulence leaving the body, and the large-scale coherent helical structure is only visible farther downstream preceding the change of decay rate. This transition, which is not observed in the wake of disks or plates, allows us to elucidate the possible role of the $|m|=1$ instability in the decay laws.

The appearance of a large-scale coherent structure in the wake and the dominance of the $|m|=1$ mode precede the non-equilibrium scaling region. This finding supports the idea that there is a connection between the presence of coherent structures and the non-equilibrium scaling of dissipation as hypothesised by Goto \& Vassilicos (2016) and more recently suggested by Alves Portela, Papadakis \& Vassilicos (2018) and Cafiero \& Vassilicos (2019). The $|m|=1$ dominance is less evident towards the end of the domain $(x / D=80)$, indicating that the wake might eventually exhibit a further transition in behaviour, possibly to the classical power law.

The present work clarifies and expands the limited available literature on slender-body wakes at high Reynolds number. Streamlined bodies with turbulent boundary layers have many hydrodynamic applications and the characterisation of their wake has significant engineering implications. This is the first study which looks into the far wake of a slender body and describes its transition to complete self-similarity. The study is performed at $R e$ in excess of any previous far-wake studies that include a wake generator, and the domain is 


\section{High-Reynolds-number wake of a slender body}

significantly longer than any previous work with this type of body. The turbulent wake of a slender body is a more complex problem than that described by classical theory and further investigation is required to answer several remaining open questions. What happens farther downstream beyond $80 \mathrm{D}$ behind the body? Is there a change so that the classic decay laws are recovered? If so, do the coherent structures subside? More simulations and experiments are needed to definitively answer these questions.

Acknowledgements. Dr K. Chongsiripinyo is thanked for useful discussions.

Funding. We gratefully acknowledge the support of Office of Naval Research Grants N00014-15-1-2718 and N00014-20-1-2253.

Declaration of interests. The authors report no conflict of interest.

\section{Author ORCIDs.}

(1) J.L. Ortiz-Tarin https://orcid.org/0000-0001-8325-9381;

() S. Nidhan https://orcid.org/0000-0003-0433-6129;

(1) S. Sarkar https://orcid.org/0000-0002-9006-3173.

\section{REFERENCES}

Alves Portela, F., Papadakis, G. \& Vassilicos, J.C. 2018 Turbulence dissipation and the role of coherent structures in the near wake of a square prism. Phys. Rev. Fluids 3 (12), 124609.

BALARAS, E. 2004 Modeling complex boundaries using an external force field on fixed Cartesian grids in large-eddy simulations. Comput. Fluids 33, 375-404.

Berger, E., Scholz, D. \& Schumm, M. 1990 Coherent vortex structures in the wake of a sphere and a circular disk at rest and under forced vibrations. J. Fluids Struct. 4 (3), 231-257.

BeVILAQUA, P.M. \& LYKOUDIS, P.S. 1978 Turbulence memory in self-preserving wakes. J. Fluid Mech. 89 (3), 589-606.

BONNIER, M. \& EIFF, O. 2002 Experimental investigation of the collapse of a turbulent wake in a stably stratified fluid. Phys. Fluids 14 (2), 791-801.

BRUCKER, K.A. \& SARKAR, S. 2010 A comparative study of self-propelled and towed wakes in a stratified fluid. J. Fluid Mech. 652, 373-404.

CAfiero, G. \& VASSILICOS, J.C. 2019 Non-equilibrium turbulence scalings and self-similarity in turbulent planar jets. Proc. R. Soc. A 475, 2225.

CANNON, S.C. 1991 Large-scale structures and the spatial evolution of wakes behind axisymmetric bluff bodies. PhD thesis, University of Arizona.

CARMOdY, T. 1964 Establishment of the wake behind a disk. Trans. ASME: J. Basic Engng 86 (4), 869-880.

CHEvray, R. 1968 The turbulent wake of a body of revolution. Trans. ASME: J. Basic Engng 90, 275-284.

CHONGSIRIPINYO, K. \& SARKAR, S. 2020 Decay of turbulent wakes behind a disk in homogeneous and stratified fluids. J. Fluid Mech. 885, A31.

Dairay, T., Obligado, M. \& Vassilicos, J.C. 2015 Non-equilibrium scaling laws in axisymmetric turbulent wakes. J. Fluid Mech. 781, 166-195.

GEORGE, W.K. 1989 The self-preservation of turbulent flows and its relation to initial conditions and coherent structures. In Advances in Turbulence (ed. W.K. George \& R. Arndt). Springer.

Gibson, C.H., Chen, C.C. \& Lin, S.C. 1968 Measurements of turbulent velocity and temperature fluctuations in the wake of a sphere. AIAA J. 6 (4), 642-649.

Goto, S. \& Vassilicos, J.C. 2016 Unsteady turbulence cascades. Phys. Rev. E 94 (5), 053108.

Gourlay, M.J, AREndT, S.C., FritTs, D.C. \& Werne, J. 2001 Numerical modeling of initially turbulent wakes with net momentum. Phys. Fluids 13 (12), 3783-3802.

Higuchi, H. \& KubOTA, T. 1990 Axisymmetric wakes behind a slender body including zero-momentum configurations. Phys. Fluids 2 (9), 1615-1623.

JimÉneZ, J.M., Hultmark, M. \& SMits, A.J. 2010 The intermediate wake of a body of revolution at high Reynolds numbers. J. Fluid Mech. 659, 516-539.

Johansson, P.B.V. \& GeORGE, W.K. $2006 a$ The far downstream evolution of the high-Reynolds-number axisymmetric wake behind a disk. Part 1. Single-point statistics. J. Fluid Mech. 555, 363-385. 


\section{J.L. Ortiz-Tarin, S. Nidhan and S. Sarkar}

Johansson, P.B.V. \& GeORGE, W.K. $2006 b$ The far downstream evolution of the high-Reynolds-number axisymmetric wake behind a disk. Part 2. Slice proper orthogonal decomposition. J. Fluid Mech. 555, 387-408.

Johansson, P.B.V., George, W.K. \& Gourlay, M.J. 2003 Equilibrium similarity, effects of initial conditions and local Reynolds number on the axisymmetric wake. Phys. Fluids 15 (3), 603-617.

KUMAR, P. \& MAHESH, K. 2018 Large-eddy simulation of flow over an axisymmetric body of revolution. J. Fluid Mech. 853, 537-563.

Monkewitz, P.A. 1988 A note on vortex shedding from axisymmetric bluff bodies. J. Fluid Mech. 192, 561-575.

Nedić, J., VAssilicos, J.C. \& GANAPATHisubramani, B. 2013 Axisymmetric turbulent wakes with new nonequilibrium similarity scalings. Phys. Rev. Lett. 111 (14), 144503.

Nicoud, F. \& Ducros, F. 1999 Subgrid-scale stress modelling based on the square of the velocity gradient tensor. Flow Turbul. Combust. 62, 183-200.

Nidhan, S., Chongsiripinyo, K., Schmidt, O.T. \& Sarkar, S. 2020 Spectral POD analysis of the turbulent wake of a disk at $R e=50000$. Phys. Rev. Fluids 5, 124606.

Obligado, M., Dairay, T. \& VAssilicos, J.C. 2016 Nonequilibrium scalings of turbulent wakes. Phys. Rev. Fluids 1 (4), 044409.

Ortiz-Tarin, J.L., Chongsiripinyo, K.C. \& Sarkar, S. 2019 Stratified flow past a prolate spheroid. Phys. Rev. Fluids 4, 094803.

Pal, A., Sarkar, S., Posa, A. \& Balaras, E. 2017 Direct numerical simulation of stratified flow past a sphere at a subcritical Reynolds number of 3700 and moderate Froude number. J. Fluid Mech. 826, 5-31.

PASQUeTTI, R. 2011 Temporal/spatial simulation of the stratified far wake of a sphere. Comput. Fluids 40 (1), 179-187.

PosA, A. \& BAlaras, E. 2016 A numerical investigation of the wake of an axisymmetric body with appendages. J. Fluid Mech. 792, 470-498.

Redford, J.A., CAStro, I.P. \& COLEMAn, G.N. 2012 On the universality of turbulent axisymmetric wakes. J. Fluid Mech. 710, 419-452.

Rigas, G., Oxlade, A.R., Morgans, A.S. \& Morrison, J.F. 2014 Low-dimensional dynamics of a turbulent axisymmetric wake. J. Fluid Mech. 755, R5.

SATO, H. \& OKADA, O. 1966 The stability and transition of an axisymmetric wake. J. Fluid Mech. 26 (2), 237-253.

Tennekes, H. \& Lumley, J.L. 1972 A First Course in Turbulence. MIT Press.

Townsend, A.A. 1976 The Structure of Turbulent Shear Flow. Cambridge University Press.

Uberoi, M.S. \& Freymuth, P. 1970 Turbulent energy balance and spectra of the axisymmetric wake. Phys. Fluids 13 (9), 2205-2210.

VAnDine, A., Chongsiripinyo, K. \& SARKAR, S. 2018 Hybrid spatially-evolving DNS model of flow past a sphere. Comput. Fluids 171, 41-52.

VAssilicos, J.C. 2015 Dissipation in turbulent flows. Annu. Rev. Fluid Mech. 47 (1), 95-114.

Wygnanski, W., Champagne, F. \& MARAsli, B. 1986 On the large-scale structures in two-dimensional, small-deficit, turbulent wakes. J. Fluid Mech. 168, 31-71.

YANG, J. \& BALARAS, E. 2006 An embedded-boundary formulation for large-eddy simulation of turbulent flows interacting with moving boundaries. J. Comput. Phys. 215 (1), 12-40. 